\title{
FMRI 3D registration based on Fourier space subsets using neural networks
}

\author{
Author(s) \\ Freire, L.C. \\ Escola Super. de Tecnol. da Saude de Lisboa, Inst. Politec. de Lisboa, Lisbon, Portugal \\ Gouveia, A.R. ; Godinho, F.M.
}

\begin{abstract}
In this work, we present a neural network (NN) based method designed for 3D rigidbody registration of FMRI time series, which relies on a limited number of Fourier coefficients of the images to be aligned. These coefficients, which are comprised in a small cubic neighborhood located at the first octant of a 3D Fourier space (including the DC component), are then fed into six NN during the learning stage. Each NN yields the estimates of a registration parameter. The proposed method was assessed for 3D rigid-body transformations, using DC neighborhoods of different sizes. The mean absolute registration errors are of approximately $0.030 \mathrm{~mm}$ in translations and 0.030 deg in rotations, for the typical motion amplitudes encountered in FMRI studies. The construction of the training set and the learning stage are fast requiring, respectively, $90 \mathrm{~s}$ and 1 to $12 \mathrm{~s}$, depending on the number of input and hidden units of the NN. We believe that $\mathrm{NN}$-based approaches to the problem of FMRI registration can be of great interest in the future. For instance, NN relying on limited $\mathrm{K}$-space data (possibly in navigation echoes) can be a valid solution to the problem of prospective (in frame) FMRI registration.
\end{abstract}

\section{Published in:}

Engineering in Medicine and Biology Society (EMBC), 2010 Annual International

Conference of the IEEE

Date of Conference: Aug. 31 2010-Sept. 42010

Page(s):

$5624-5627$

ISSN :

1557-170X

Print ISBN:

978-1-4244-4123-5

INSPEC Accession Number:

11650555

Conference Location : 
Buenos Aires

Digital Object Identifier :

10.1109/IEMBS.2010.5628038

PubMed ID :

21097303

\section{Keywords}

IEEE TERMS

- Artificial neural networks

- Biomedical imaging

- Image registration

- Solid modeling

- Three dimensional displays

- Time series analysis

- Training

\section{MeSH TERMS}

- Computer Simulation

- Fourier Analysis

- Image Interpretation, Computer-Assisted

- Imaging, Three-Dimensional

- Magnetic Resonance Imaging

- Neural Networks (Computer)

- $\quad$ Time Factors 